\title{
Decreasing severe pain and serious adverse events while moving intensive care unit patients: a prospective interventional study (the NURSE-DO project)
}

Audrey de Jong ${ }^{1}$, Nicolas Molinari ${ }^{2}$, Sylvie de Lattre ${ }^{1}$, Claudine Gniadek', Julie Carr ${ }^{1}$, Mathieu Conseil ${ }^{1}$, Marie-Pierre Susbielles ${ }^{1}$, Boris Jung ${ }^{1,3}$, Samir Jaber ${ }^{1,3}$ and Gérald Chanques ${ }^{1,3^{*}}$

\begin{abstract}
Introduction: A quality-improvement project was conducted to reduce severe pain and stress-related events while moving ICU-patients.

Methods: The Plan-Do-Check-Adjust cycle was studied during four one-month phases, separated by five-month interphases. All consecutive patients staying more than 24 hours were evaluated every morning while being moved for nursing care (bathing, massage, sheet-change, repositioning). Phase 1 was considered as the baseline. Implemented and adjusted quality-interventions were assessed at phases 2 and 3, respectively. An independent post-intervention controlaudit was performed at Phase 4. Primary-endpoints were the incidence of severe pain defined by a behavioral pain scale $>$ 5 or a 0 to 10 visual numeric rating scale $>6$, and the incidence of serious adverse events (SAE): cardiac arrest, arrhythmias, tachycardia, bradycardia, hypertension, hypotension, desaturation, bradypnea or ventilatory distress. Pain, SAE, patients' characteristics and analgesia were compared among the phases by a multivariate mixed-effects model for repeatedmeasurements, adjusted on severity index, age, admission type (medical/surgical), intubation and sedation status.

Results: During the four studied phases, 630 care procedures were analyzed in 53, 47, 43 and 50 patients, respectively. Incidence of severe pain decreased significantly from 16\% (baseline) to $6 \%$ in Phase 3 (odds ratio (OR) $=0.33(0.11 ; 0.98), P=0.04)$ and $2 \%$ in Phase $4(\mathrm{OR}=0.30(0.12 ; 0.95), P=0.02)$. Incidence of SAE decreased significantly from $37 \%$ (baseline) to $17 \%$ in Phase 3 and $21 \%$ in Phase 4 . In multivariate analysis, SAE were independently associated with Phase $3(\mathrm{OR}=0.40(0.23 ; 0.72), P<0.01)$, Phase $4(\mathrm{OR}=0.53(0.30 ; 0.92), P=0.03)$, intubation status $(\mathrm{OR}=1.91(1.28 ; 2.85), P<0.01)$ and severe pain $(\mathrm{OR}=2.74(1.54 ; 4.89), P<0.001)$.

Conclusions: Severe pain and serious adverse events are common and strongly associated while moving ICU patients for nursing procedures. Quality improvement of pain management is associated with a decrease of serious adverse events. Careful documentation of pain management during mobilization for nursing procedures could be implemented as a health quality indicator in the ICU.
\end{abstract}

\section{Introduction}

Pain is a frequent event in intensive care unit (ICU) patients, with an incidence of moderate to severe pain during the ICU stay of up to $50 \%$ in medical as well as surgical patients [1-3]. Pain is associated with acute

\footnotetext{
*Correspondence: g-chanques@chu-montpellier.fr

'Intensive Care and Anesthesiology Department, University of Montpellier Saint Eloi Hospital, 80 Avenue Augustin Fliche, Montpellier, 34295, France Full list of author information is available at the end of the article
}

stress response including changes in heart rate, blood pressure, respiratory rate, neuro-endocrine secretion and psychological distress, such as agitation $[4,5]$. It has recently been reported that improved pain management was associated with improved patient outcome in the ICU [1,6-8]. However, pain remains currently underevaluated and under-treated [3,9-12]. Therefore, pain management is highly challenging in the ICU setting.

\section{Biomed Central}

(C) 2013 de Jong et al.; licensee BioMed Central Ltd. This is an open access article distributed under the terms of the Creative Commons Attribution License (http://creativecommons.org/licenses/by/2.0), which permits unrestricted use, distribution, and reproduction in any medium, provided the original work is properly cited. 
One of the most common painful procedures in ICU patients is moving and turning for nursing care procedures (bathing, massage of back and pressure points, sheets change, repositioning) $[3,13]$. Pain during the first turning of the day is especially challenging to manage in our ICU. Indeed, this is often the longest turning time and includes the highest number of mobilizations and nursing care procedures. Moreover, the early morning nurses often have to manage ICU patients in collaboration with a reduced medical night-shift staffing, leading to necessarily greater nurse autonomy [14]. For instance, it has been reported for the past decade that between $50 \%$ of patients in the USA [9] and $80 \%$ in Europe $[3,15]$ received no extra medication even though pain intensity increased during that procedure. More recently, a study assessing 330 turnings in 96 medical-surgical patients reported that the pain score significantly increased between rest and turning, while a bolus of analgesic was used in less than $15 \%$ of the turnings [16]. Moreover, serious adverse events (SAE) related to moving complex ICU patients are poorly documented. These SAE could be determined by the mobilization itself and/or the stress response associated with pain.

The present study was conducted to test the hypothesis that the implementation of a quality improvement process for pain management while moving ICU patients would be associated with a decreased incidence of both severe pain and SAE, and that those SAE would often be associated with pain events.

\section{Materials and methods Population}

The study took place in the 16-bed medical-surgical ICU of St Eloi Hospital, a 660-bed teaching and referral facility of the University of Montpellier in France, staffed by 35 registered nurses (RNs), 25 nurse assistants, 3 certified registered nurse anesthetists, 7 attending physicians and 4 residents. Nurse to patient ratio was $1: 2.5$ as required in France [17]. The ICU has 24-hour anesthesiologist/intensivist medical staffing including three anesthesia residents and three attendings on dayshift, one resident and one attending on nightshift. RNs systematically and routinely assess pain and agitation at rest and during procedures using dedicated tools validated for ICU patients since 2003 [1]. For patients receiving a continuous infusion of sedatives, RNs have been using a sedation-analgesia algorithm since 2007 [18]. In the absence of continuous sedation, or previous analgesic ordering, a medical doctor was called in case of any pain or agitation events [1].

All consecutive patients $\geq 18$ yrs old and staying in the ICU for more than $24 \mathrm{hrs}$ were eligible. Exclusion criteria were decision to withdraw life-support within $48 \mathrm{hrs}$ after admission and lacking data. Because of the observational, non-invasive design of this quality-improvement study based on the Plan-Do-Check-Adjust method which aimed to apply recommended practice guidelines [19], the need for written consent was waived as for previous published quality studies on sedation-analgesia practices in ICU patients [20] by the local scientific and ethics committee of Comité d'Organisation et de Gestion de l'Anesthésie Réanimation du Centre Hospitalier Universitaire de Montpellier (COGAR), which approved the conduct of the study.

\section{Study design \\ "Plan-step": multidisciplinary ICU work-group and choice of the studied procedure}

A multidisciplinary work-group was created, composed of three registered nurses, three assistant nurses, and three physicians (two attending physicians and one resident). All members received institutional education provided by the Hospital Pain Committee. Five meetings were necessary to elaborate the quality study design. The first nursing care procedure in the morning was chosen to be studied because it accounts in our ICU for the care which requires the longest duration of turning, including the largest number of moves and nursing care procedures in the day (bathing, massage of back and pressure points, sheet changing, repositioning, frequent change of dressings and placement of stockings and foot splints). Also, the work group had the impression that there was a strong contrast between the end and beginning of the day regarding pain, agitation and the number of alarms ringing from monitoring systems early in the morning. Contrary to pain at rest, pain during procedures was rarely reported in medical charts. We made the hypothesis that managing procedural pain during the first turning of the day would be the most challenging in our ICU. Figure 1 represents the study design that included four one-month studied phases separated by interphase periods of four to six months, according to the Plan-Do-Check-Adjust method [20-22]. Total length of the study was 20 months. The present quality improvement process was the third quality process performed in the ICU regarding the management of sedation and analgesia. The first quality improvement process, aimed at implementing a systematic assessment of pain and agitation in the ICU using validated tools, was initiated in 2002 and evaluated in 2003 [1]. The second project (2006 to 2007) was aimed at evaluating nurse interventions regarding a sedation-analgesia algorithm and at comparing them to a North American ICU [18].

\section{"Do- step -A": studied phase-1 (February 2010)}

Every first turning of the day, between 6 and 8 AM was evaluated (see below, evaluated parameters).

During this phase, a de-identified questionnaire was given to every $\mathrm{RN}$ and nurse assistant in order to assess their knowledge of written guidelines regarding sedationanalgesia in the ICU and their difficulties in managing sedation-analgesia routinely. 


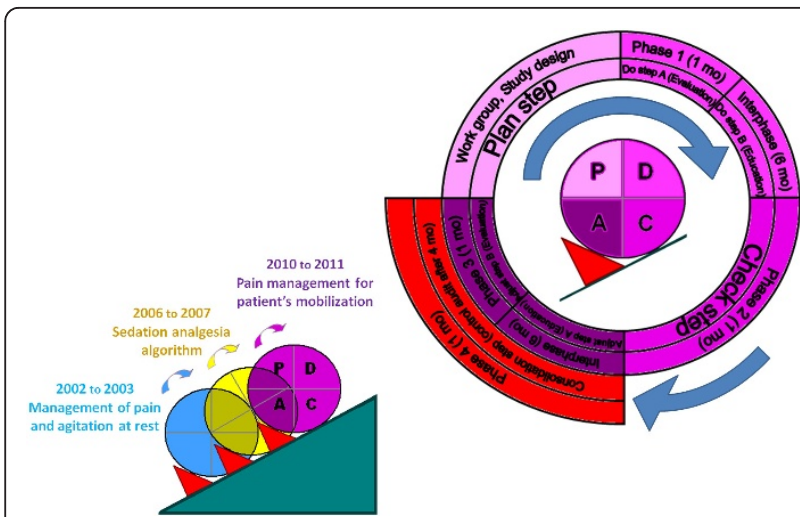

Figure 1 Study-design and quality method. This figure represents the quality-improvement process of pain and serious adverse events while moving ICU patients for turning and nursing care procedures. This 20-month process following the P-D-C-A steps was evaluated by four one-month studied phases separated by inter-study phases of four to six months. The present quality improvement process was the third quality process performed in the ICU regarding the management of sedation and analgesia. Consecutive improvement steps were followed according to the Plan-Do-Check-Adjust method for quality-improvement: - P (Plan-step): Multidisciplinary ICU work group creation, choice of the studied procedure and design of the quality improvement process. - D (Do-step): Beginning of the NurseDo study by a one-month baseline evaluation of pain management by nurse while moving the patients (studied Phase 1). Educational interventions for optimized pain management by nurse (Nurse-Do) started after the baseline studied phase. - C (Check step): Onemonth evaluation (Check) of educational interventions (studied Phase 2). - A (Adjust step): Adjustment of educational interventions implicating an increased multidisciplinary team collaboration, onemonth evaluation (Check) of adjusted interventions (studied Phase 3). - Consolidation step: one-month control audit of the PDCA quality improvement process (studied Phase 4).

"Do-step B": first inter-study phase (March to August 2010) Based on Phase 1 and questionnaire results, educational interventions were planned and educational posters were constructed and posted. Educational intervention was provided for all the nursing and medical staff by members of the work group during scheduled courses intended for 5 to 10 staff members at a time. Educational objectives included the ability: 1) to assess and control pain at rest before any moving procedure, 2) to determine the duration of peak analgesia for analgesic drugs used in the ICU in order to anticipate administration of drugs before moving a patient, 3) to escalate analgesic drugs according to the World Health Organization's analgesic steps in case of ineffectiveness after having referred to previous pain assessment, 4) to administer music therapy as well as other non-pharmacological analgesia therapies. Analgesics were selected by physicians according to the clinical situation and administered by RNs. Non-pharmacological therapies were selected and administered by the RN only. In order to develop the use of non-pharmacological therapies, headphones and dedicated pieces of music therapy were implemented in every patient's room. Music scores were composed by music therapists. The main characteristics of music (tempo, intensity, number of instruments) progressively decreased, then stabilized to a low pattern (slow tempo, low level of sound, one or two instruments), and finally increased slowly before removing the headphones from the patient. In other words, music characteristics followed a U-shape. Total duration of a music therapy session was $40 \pm 5$ minutes. Nurses and physicians were specifically educated by a music therapist during this interphase.

Finally, the clinical information system software was modified to include specificities of pain management for nursing care procedures. Posters referring to pain management and the sedation-analgesia algorithm were created to highlight educational objectives previously described. Posters were posted in every patient's room. These posters are shown in electronic supplement in their original French version as well as an English version (see Additional files 1, 2, 3, 4).

\section{"Check-step": studied Phase 2 (September 2010)}

Every first turning of the day, between 6 and 8 AM was evaluated (see below, Evaluated parameters). This phase was aimed to measure the impact of the educational interventions.

\section{"Adjust- step A": second inter-study phase (October 2010 to} March 2011)

During six months (October 2010 to March 2011) a data and problems analysis was performed and multidisciplinary medical and nursing strategy was adjusted. As from this moment, medical staff was asked to systematically order one or more analgesics to be administered early in the morning before the nursing care procedures. Nurses had the possibility of using one or more of these analgesic drugs according to their discretion based on pain assessments. Moreover, pain management for the nursing procedure was standardized and systematically checked along with other nursing issues during daily medical rounds. Compliance with the quality improvement project was corrected by reminders and analysis of specific situations by the nurse manager and the ICU medical director during their weekly nursing medical round.

\section{"Adjust-step B": studied Phase 3 (April 2011)}

Every first turning of the day, between 6 and 8 AM was evaluated (see below, evaluated parameters). This phase was aimed to measure the impact of adjustments made during the second interphase.

\section{"Consolidation- step": studied Phase 4 (September 2011)}

A consolidation step was added to the PDCA-cycle to measure sustained quality-improvement as the new standard [21,23]. Therefore, a control-audit was realized by an independent observer, four months after the end of the study. All eligible patients were consecutively included. Choice of evaluated moving and nursing procedures was 
made by randomization using a random number generation method.

\section{Evaluated parameters \\ Studied phases 1, 2, 3}

1) Pain was measured by the bedside $R N$ while the patient was at rest before and during any moving procedures routinely throughout the study process, using validated ICU pain tools. Communicating patients rated their discomfort intensity on the visually enlarged numeric rating scale (NRS) from 0 (no pain) to 10 (maximum imaginable discomfort) [24]. For non-communicating patients (sedated or delirious patients), pain was assessed by nurses using the behavioral pain scale (BPS) for intubated patients [25] and the non-intubated BPS (BPS-NI) for nonintubated patients [26]. Severe pain events were defined by a NRS level $>6$ according to the usual definition [27] or a BPS/BPS-NI score $>5$ according to validation studies $[25,26,28]$. Those studies demonstrated a score $>5$ for procedures known as very painful. Moderate pain was defined by a NRS level from 4 to 6 or a BPS $>3$ (minimal score) but $<6$. Awareness was assessed at baseline by the Richmond Agitation Sedation Scale (RASS) [29]. Interrater reliability of these sedation and pain scales has been assessed repeatedly in the ICU $[1,18,26,30]$. All bedside RNs present during the study phases were fully familiar with using these pain and sedation scales routinely, for both sedated and non-sedated patients.

2) SAE related to acute stress-response were assessed by physiological parameters (cardiac rhythm, heart rate, mean arterial pressure, respiratory rate and oximetry), measured continuously by the ICU monitor and recorded before and while the moving procedure by the bedside RN on a sheet dedicated to the study. SAE were defined as cardiac arrest, a new arrhythmia event and clinically relevant changes before and during the procedure defined as follows:

- Tachycardia: heart rate $\geq 110$ beats/minute $(b / \mathrm{min})$ if $<100 \mathrm{~b} /$ min before the procedure

- Bradycardia: heart rate $\leq 60 \mathrm{~b} / \mathrm{min}$ if $>70 \mathrm{~b} / \mathrm{min}$ before

- Hypertension: mean arterial pressure $\geq 110 \mathrm{mmHg}$ if $<100 \mathrm{mmHg}$ before

- Hypotension: mean arterial pressure $\leq 65 \mathrm{mmHg}$ if $>$ $70 \mathrm{mmHg}$ before

- Desaturation: oxygen saturation $\leq 90 \%$ if $>92 \%$ before

- Bradypnea: respiratory rate $\leq 10 \mathrm{c} / \mathrm{min}$ if $>10 \mathrm{c} / \mathrm{min}$ before

- Ventilatory distress: severe ventilator asynchrony (nonstop coughing or impossible ventilation) in mechanically ventilated patients and/or tachypnea (respiratory rate $\geq 35 \mathrm{c} / \mathrm{min}$ if it was $<35 \mathrm{c} / \mathrm{min}$ )
3) Pharmacological therapies given within four hours prior to the moving procedure were reported by the bedside nurse on the patient flow sheet. Non-pharmacological therapies (explanation of the nursing care procedure, therapeutic massage, music, music therapy) performed to decrease pain while being moved were reported by the bedside nurse on a sheet dedicated to the study.

4) Demographic and medical data were prospectively recorded. Age, gender, type of admission (medical or surgical) and Simplified Acute Physiological Score (SAPS) II [31] were collected within $24 \mathrm{hrs}$ after ICU admission. Medical admission was defined by the absence of surgical intervention within seven days prior to ICU admission.

\section{Studied Phase 4 (control audit)}

Pain was measured by bedside nurses at rest before and during any moving procedures, similarly to the other phases but reported routinely on the patient's flow sheet instead of a dedicated study sheet. Physiological parameters were recorded every 30 minutes by the patient's Clinical Information System (ICIP-Carevue, PhilipsMedical-Systems, Eindhoven, The Netherlands). Maximal or minimal values, which had been recorded an hour before and after the moving procedure, were analyzed. Pharmacological therapies were evaluated as for the other phases. Non-pharmacological therapies were not assessed because of the absence of systematic notification in the medical chart.

\section{Endpoints}

The primary endpoints were the incidence of severe pain defined by the proportion of patients who developed a severe pain event (BPS $>5$ and/or NRS $>6$ ) and the incidence of SAE defined by the proportion of patients who developed at least one SAE while being moved. Secondary endpoints were incidence of moderate pain, the existence of a relationship between pain and SAE, and a change in analgesic ordering practice patterns.

\section{Statistical analysis}

Based on previous data [24], an incidence of severe pain of $26 \%$ was observed in our ICU during mobilization for nursing care procedures. To show a $50 \%$ reduction of severe pain, $n=100$ procedures needed to be analyzed for every phase, with alpha 0.05 and beta 0.10 . Missing data were expected because bedside RNs would sometimes forget or not have enough time to fill in the sheets dedicated for the study due to an eventual high workload in the ICU. Taking into account missing data and the rate of empty rooms in the ICU at a given time, this meant enrolling consecutive patients hospitalized in the ICU within one month for every phase. Also, repeated one-month phases could allow for implementing the study effect (Hawthorne effect) into 
a routine process which was part of the quality-improvement project [32]. Reference (baseline) phase was Phase 1.

Quantitative data were shown as mean and standard deviation or median and $25^{\text {th }}$ to $75^{\text {th }}$ percentiles according to data distribution. Student $t$-test or Wilcoxon test (quantitative data) and chi-square test (qualitative data) were used to compare patients included in the four phases. Because moving for nursing care procedures was evaluated every day of the ICU stay, one patient could be evaluated several times (repeated measures).

Thus, pain events, serious adverse events and analgesic ordering were compared in univariate analysis using a generalized linear mixed-effects model for repeated measures, taking into account repeated measures as random variables. Multivariate analysis of pain events and SAE was secondly performed using a generalized linear mixed-effects model for repeated measures. Variables were selected if $P$-value was less than 0.20 in the univariate analysis and a stepwise procedure was used to select the final model. Furthermore, a sensitivity analysis was performed, removing tachycardia and hypertension, which are common events associated with pain, from the definition of SAE. This was done to measure the impact of the quality project on the incidence of other SAE. A $P$-value of $\leq .05$ was considered statistically significant. Data were analyzed by a senior statistician from the Department of Statistics of the University of Montpellier Hospital using the R.2.13.0 software.

\section{Results}

\section{Results from the questionnaire regarding sedation/} analgesia practices

Among the nursing staff, 21 (60\%) RNs and 17 (68\%) nurse assistants answered the questionnaire during Phase 1. Pain assessment tools were thought to be adapted to ICU patients by all 21 (100\%) RNs. Before the study, 17 (71\%) RNs had already experienced a disagreement with doctors regarding pain management and 5 (29\%) nurse assistants had experienced a disagreement with RNs. Disagreements occurred because some patients could have been in pain but physicians or nurses did not allow for increasing analgesics because of the risk of developing side-effects. Fourteen (58\%) RNs did not refer to patients' previous pain assessments and analgesia documentation to better adjust analgesia for nursing care procedures for a given patient. Among the 21 RNs, 9 (43\%) desired more autonomy in pain management. A greater autonomy was achieved in the quality improvement project by allowing nurses to administer selected analgesics. Because almost half of the nurses did not want greater autonomy, analgesic choice remained the physicians' role and pain management was developed more collaboratively between nurses and physicians. Educational interventions aimed at decreasing the incidence of severe pain and SAE and improving analgesics ordering were evaluated during the four studied phases.

\section{Evaluation of the quality improvement project across the four studied phases}

Overall 630 procedures were analyzed in 193 patients during the four studied phases, in 53, 47, 43 and 50 patients, respectively. The flow chart of the study is shown in Figure 2. Table 1 summarizes patients' demographic and medical characteristics. No significant difference was shown across groups except in Phase 3 during which patients had a significantly lower rate of procedures evaluated while receiving a continuous infusion of sedatives (propofol or midazolam).

Incidence of severe pain, as well as at least one SAE (cardiac arrest, arrhythmias, tachycardia, bradycardia, hypertension, hypotension, desaturation, bradypnea or ventilatory distress), decreased over the quality improvement study, while the proportion of analgesia given for nursing care procedures increased (Figure 3 ). The difference was not significant between Phase 1 (baseline) and Phase 2 (first intervention P-D-C-A step) but became significant during Phase 3 (adjusted-intervention P-D-C-A step) and Phase 4 (consolidation P-D-C-A step).

In multivariate analysis adjusted for cofactors and repeated measures (Table 2), severe pain was significantly less frequent during both Phase 3 (odds ratio $(\mathrm{OR})=0.33(0.11 ; 0.98), P=0.04)$ and Phase $4(\mathrm{OR}=$ 0.30 (0.12; 0.95), $P=0.02$ ). Incidence of moderate pain did not significantly decrease during the study (see Additional file 5, Table S1).

A lower incidence of SAE was independently associated with Phase $3(\mathrm{OR}=0.40(0.23 ; 0.72), P<0.01)$ and Phase $4(\mathrm{OR}=0.53(0.30 ; 0.92), P=0.03)$ whereas a higher incidence of SAE was associated with intubated status $(\mathrm{OR}=1.91(1.28 ; 2.85), P<0.01)$ and severe-pain $(\mathrm{OR}=2.74(1.54 ; 4.89), P<0.001)$ (Table 3). Incidence of SAE was not associated with moderate pain. Detailed incidence of SAE is shown in Table 4 . The sensitivity analysis showed that the incidence of at least one SAE (not taking into account tachycardia and/or hypertension) was also associated with Phase 3, Phase 4, intubation status and severe-pain (see Additional file 5, Table S2). Finally, hypotension was a little more frequent during Phase 4 but there was no significant association among hypotension, studied phases and analgesia $(P=0.60$, mixed-effect model).

There was a change in analgesic ordering practice patterns across the quality improvement project (Table 5). Use of tramadol was significantly higher in Phase 3 and in Phase 4 than in Phase 1. Administration of at least one analgesic drug was significantly higher in Phase 3 and in Phase 4. New non-pharmacological therapies 


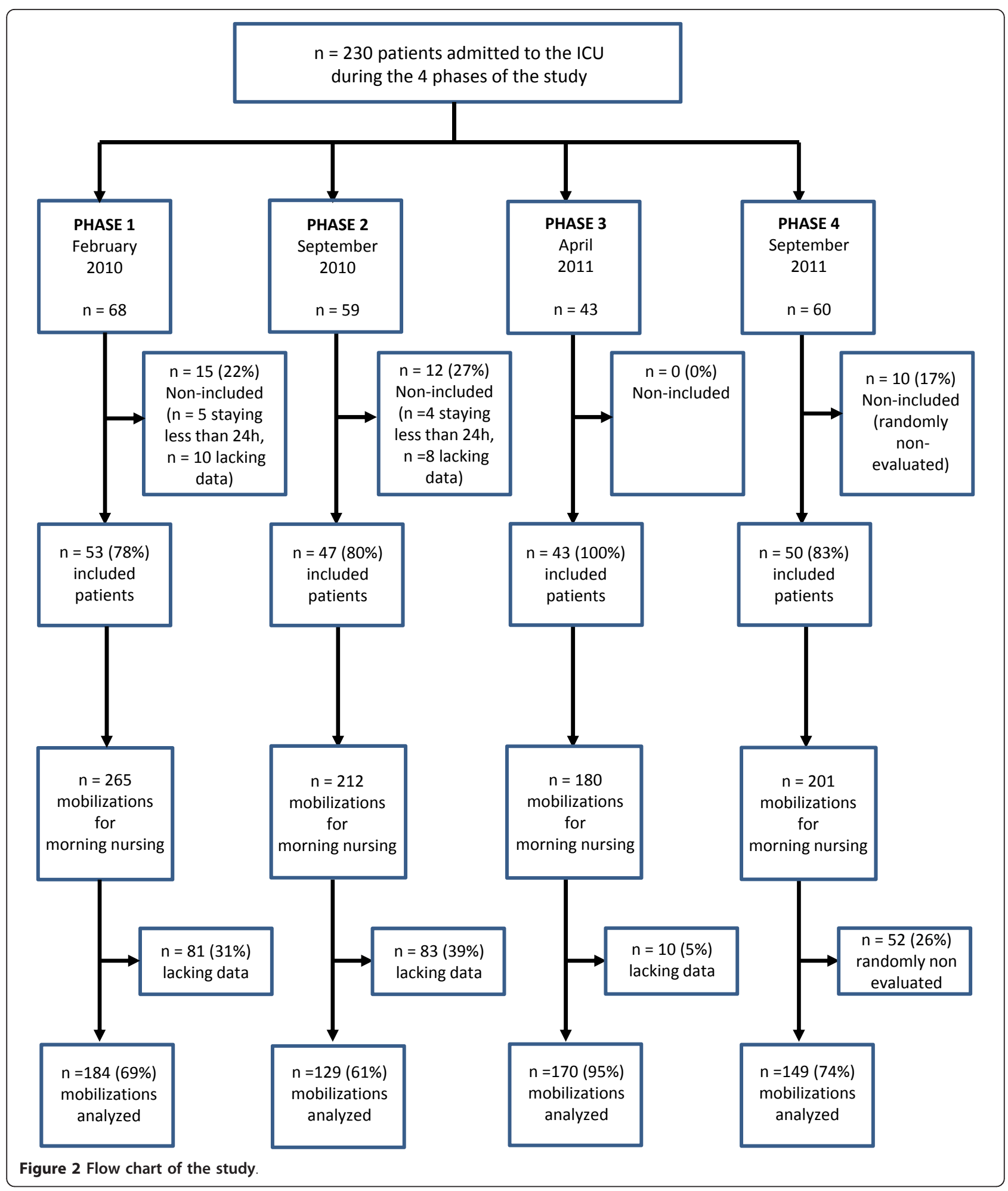

were implemented in the study, such as music-therapy, which was displayed in each patient's room with dedicated headphones and music scores specifically composed for relaxation. However, if music therapy and the total number of non-pharmacological therapies used to treat pain significantly increased between Phase 1 and Phase 2, this increase was not sustained afterward (Table 5). 
Table 1 Characteristics of patients included in the four phases of the study.

\begin{tabular}{|c|c|c|c|c|c|c|c|}
\hline & $\begin{array}{c}\text { Phase } 1 \\
\text { (baseline) } \\
n=53\end{array}$ & $\begin{array}{c}\text { Phase } 2 \\
\text { (intervention) } \\
n=47\end{array}$ & $\begin{array}{c}\text { Phase } 3 \\
\text { (adjustment) } \\
n=43\end{array}$ & $\begin{array}{c}\text { Phase } 4 \\
\text { (consolidation) } \\
n=50\end{array}$ & $\begin{array}{c}P \\
1-2\end{array}$ & $\begin{array}{c}P \\
1-3\end{array}$ & $\begin{array}{c}P \\
1-4\end{array}$ \\
\hline Age (years), median (IQR) & $64(54 ; 74)$ & $65(49 ; 74)$ & $61(49 ; 67)$ & $61(51 ; 69)$ & 0.87 & 0.10 & 0.24 \\
\hline Female Sex, n (\%) & $19(36 \%)$ & $18(38 \%)$ & $12(28 \%)$ & $13(26 \%)$ & 0.84 & 0.51 & 0.30 \\
\hline SAPS II, median (IQR) & $41(31 ; 54)$ & $38(27 ; 53)$ & $34(27 ; 41)$ & $37(26 ; 53)$ & 0.64 & 0.07 & 0.57 \\
\hline Surgical admission*, n (\%) & $22(42 \%)$ & $25(53 \%)$ & $16(37 \%)$ & $26(52 \%)$ & 0.32 & 0.29 & 0.33 \\
\hline Mechanical ventilation, n (\%) & $24(45 \%)$ & $21(45 \%)$ & $13(30 \%)$ & $22(45 \%)$ & 0.95 & 0.13 & 0.90 \\
\hline Sustained use of sedatives, n (\%) & $22(42 \%)$ & $15(32 \%)$ & $9(21 \%)$ & $14(29 \%)$ & 0.32 & 0.03 & 0.15 \\
\hline RASS level, median (IQR) & $0(-3 ; 0)$ & $0(-1 ; 0)$ & $0(0 ; 0)$ & $0(-1 ; 0)$ & 0.92 & 0.52 & 0.41 \\
\hline Number of procedures evaluated per patient, median (IQR) & $2(1 ; 4)$ & $2(1 ; 3)$ & $3(1 ; 5)$ & $3(3 ; 3)$ & 0.39 & 0.38 & 0.24 \\
\hline
\end{tabular}

IQR, Inter-Quartile-Range ( $25^{\text {th }}$ to $75^{\text {th }}$ percentiles); RASS, Richmond-Agitation-Sedation-Scale [29] from -5 (deep sedation) to +4 (combative agitation), a level of 0 defines an awake state of awareness without any agitation; SAPS II, Simplified-Acute-Physiology-Score II [31]. * Surgical patients all underwent abdominal surgery.

\section{Discussion}

The main findings of this quality improvement project are that moving an ICU patient for nursing care procedures is associated with severe adverse events (SAE) in one out of three procedures. The incidence of at least one SAE (cardiac arrest, arrhythmias, tachycardia, bradycardia, hypertension, hypotension, desaturation, bradypnea or ventilatory distress) is strongly associated with severe pain in multivariate analysis. A healthcare quality improvement project of pain management, while moving
ICU patients, is associated with a decrease in both severe pain and SAE.

Being moved for nursing care procedures is one of the most painful procedures experienced by the patient during the ICU stay, whatever the type of admission (medical, surgical or trauma) [3,13,16,33]. Nevertheless, except for trauma and surgical patients, moving is currently not considered a painful procedure by ICU healthcare workers and physicians [34]. Similarly, to our knowledge, no study has reported yet whether pain

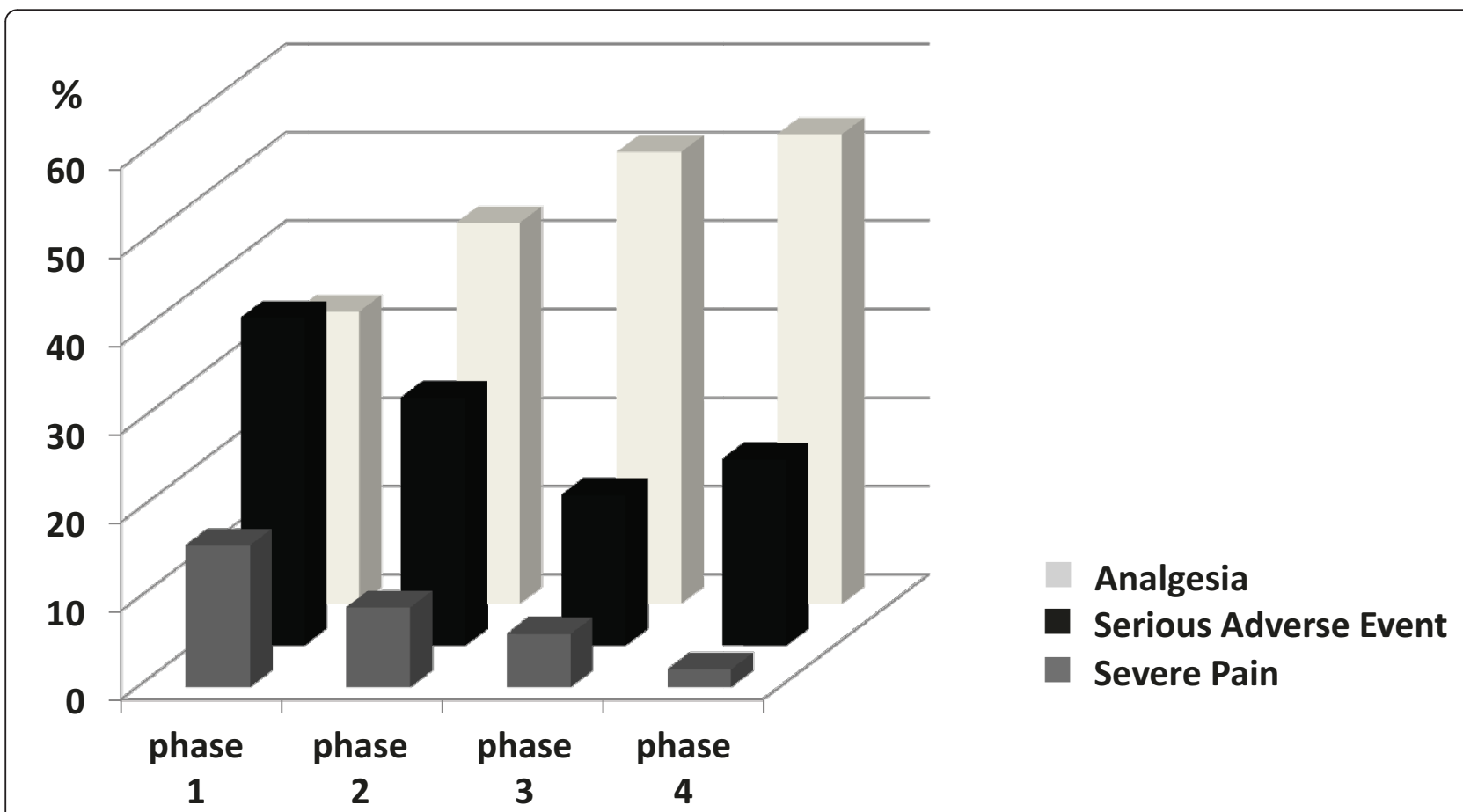

Figure 3 Incidence of severe pain, serious adverse events and analgesia. This figure shows that the incidence of severe pain and serious adverse events (SAE) decreased across the quality improvement study while the proportion of given analgesia increased. The difference was significant for severe pain $(P=0.04$ and 0.02$)$, SAE $(P<0.001$ and $P<0.01)$ and analgesia $(P=0.01$ and $P<0.01)$ between Phase 1 (baseline) and Phases 3 and 4 , respectively. 
Table 2 Factors associated with severe-pain determined by univariate and multivariate mixed-effects model analysis

\begin{tabular}{|c|c|c|c|c|c|c|}
\hline & \multicolumn{4}{|c|}{ Univariate analysis } & \multicolumn{2}{|c|}{ Multivariate analysis } \\
\hline & $\begin{array}{c}\text { All } \\
\text { procedures } \\
(n=632)\end{array}$ & $\begin{array}{l}\text { Severe pain } \\
(n=61)\end{array}$ & $\begin{array}{l}\text { Others } \\
(n=571)\end{array}$ & $P$ & $\begin{array}{c}\text { OR } \\
(95 \% \mathrm{Cl})\end{array}$ & $P$ \\
\hline Phase 1, n (\%) & 184 & $30(49 \%)$ & $154(27 \%)$ & & & \\
\hline Phase 2, n (\%) & 129 & $12(20 \%)$ & $117(20 \%)$ & 0.22 & & \\
\hline Phase 3, n (\%) & 170 & $11(18 \%)$ & $159(28 \%)$ & 0.04 & $0.33(0.11 ; 0.98)$ & 0.04 \\
\hline Phase 4, n (\%) & 149 & $8(13 \%)$ & $141(25 \%)$ & 0.03 & $0.30(0.12 ; 0.95)$ & 0.02 \\
\hline Age, median (IQR) & $63(51 ; 71)$ & $64(57 ; 76)$ & $63(51 ; 71)$ & 0.16 & & \\
\hline Female gender, $\mathrm{n}(\%)$ & $186(29 \%)$ & $17(28 \%)$ & 169 (30\%) & 0.94 & & \\
\hline SAPS II, median (IQR) & $39(29 ; 41)$ & $39(27 ; 51)$ & $39(30 ; 51)$ & 0.48 & & \\
\hline Surgical admission, n (\%) & $219(35 \%)$ & $25(41 \%)$ & 194 (34\%) & 0.23 & & \\
\hline Intubation status, n (\%) & $216(34 \%)$ & $24(39 \%)$ & $192(34 \%)$ & 0.95 & & \\
\hline Sustained use of sedatives, n (\%) & $114(18 \%)$ & $10(16 \%)$ & $104(18 \%)$ & 0.69 & & \\
\hline RASS level, median (IQR) & $0(-1 ; 0)$ & $0(-1 ; 0)$ & $0(-1 ; 0)$ & 0.31 & & \\
\hline
\end{tabular}

Cl, Confidence-Interval; IQR, Inter-Quartile-Range ( $25^{\text {th }}$ to $75^{\text {th }}$ percentiles); OR, Odd-Ratio; RASS, Richmond-Agitation-Sedation-Scale [29]; SAPS II, Simplified-AcutePhysiology-Score II [31]. In addition to studied phases, variables were selected in multivariate analysis if $P$-value was less than 0.20 in the univariate analysis, that is, were included in the final mixed-effect model: studied phases and age.

might be a barrier for active mobilization in ICU patients and if a specific analgesia given to decrease pain while moving ICU patients would be associated with a greater chance to achieve rehabilitation objectives in the ICU setting $[35,36]$.

One of the reasons not to treat pain is that ICU physicians may be uncomfortable ordering analgesic drugs [37] because of frequent organ dysfunction, altered pharmacokinetics and pharmacodynamics, and impaired mental status in critically ill patients [38]. Indeed, adverse events have been reported in critically ill patients even with non-opioid WHO's step-1 analgesics, such as acetaminophen [39] and nefopam [40]. In the present study, analgesics were administered upon nurse discretion but were chosen among eligible analgesics ordered by physicians according to the context and for each patient. Decreased incidence of severe pain and increased rate of analgesic administration observed during adjusted and consolidated steps of the quality project suggests that collaboration between nurses and physicians, which was the aim of educational intervention at the adjusted step, improved regarding

Table 3 Factors associated with serious adverse events determined by univariate and multivariate mixed-effects model analysis

\begin{tabular}{|c|c|c|c|c|c|c|}
\hline & \multicolumn{4}{|c|}{ Univariate analysis } & \multicolumn{2}{|c|}{ Multivariate analysis } \\
\hline & $\begin{array}{l}\text { All procedures } \\
(n=632)\end{array}$ & $\begin{array}{c}\text { Serious adverse events } \\
\text { YES } \\
(n=164)\end{array}$ & $\begin{array}{c}\text { Serious adverse events } \\
\text { NO } \\
(n=468)\end{array}$ & $P$ & $\begin{array}{c}\text { OR } \\
(95 \% \mathrm{Cl})\end{array}$ & $P$ \\
\hline Phase 1, n (\%) & $184(29 \%)$ & $68(41 \%)$ & $116(25 \%)$ & & & \\
\hline Phase 2, n (\%) & $129(20 \%)$ & $36(22 \%)$ & $93(20 \%)$ & 0.09 & & \\
\hline Phase 3, n (\%) & $170(27 \%)$ & $29(18 \%)$ & $141(30 \%)$ & $<0.001$ & $0.40(0.23 ; 0.72)$ & $<0.01$ \\
\hline Phase 4, n (\%) & $149(24 \%)$ & $31(19 \%)$ & $118(25 \%)$ & $<0.01$ & $0.53(0.30 ; 0.92)$ & 0.03 \\
\hline Age, median (IQR) & $63(51 ; 71)$ & $64(56 ; 75)$ & $62(51 ; 70)$ & 0.09 & & \\
\hline Female gender, $\mathrm{n}(\%)$ & $186(29 \%)$ & $44(27 \%)$ & $142(30 \%)$ & 0.44 & & \\
\hline SAPS II, median (IQR) & $39(29 ; 41)$ & $39(31 ; 53)$ & $38(28 ; 50)$ & 0.19 & & \\
\hline Surgical admission, n (\%) & $219(35 \%)$ & $104(63 \%)$ & 309 (66\%) & 0.54 & & \\
\hline Intubation status, n (\%) & $216(34 \%)$ & $79(48 \%)$ & 137 (29\%) & $<0.01$ & $1.91(1.28 ; 2.85)$ & $<0.01$ \\
\hline Sustained use of sedatives, n (\%) & $114(18 \%)$ & $38(23 \%)$ & $76(16 \%)$ & 0.17 & & \\
\hline RASS level, median (IQR) & $0(-1 ; 0)$ & $0(-2 ; 0)$ & $0(-1 ; 0)$ & 0.05 & & \\
\hline \multicolumn{7}{|l|}{ Pain during moving } \\
\hline Moderate pain, n (\%) & $160(25 \%)$ & $41(25 \%)$ & $119(25 \%)$ & 0.81 & & \\
\hline Severe pain, n (\%) & $61(10 \%)$ & $30(18 \%)$ & $31(7 \%)$ & $<0.001$ & $2.74(1.54 ; 4.89)$ & $<0.001$ \\
\hline
\end{tabular}

$\mathrm{Cl}$, Confidence interval; IQR, Interquartile Range ( $25^{\text {th }}$ to $75^{\text {th }}$ percentiles); OR, Odds ratio; RASS, Richmond Agitation-Sedation Scale [29]; SAPS II, Simplified Acute Physiology Score II [31]. In addition to studied phases, variables were selected in multivariate-analysis if $P$-value was less than 0.20 in the univariate analysis, that is, were included in the final mixed-effect model: studied phases, age, SAPS II, intubation status, sustained use of sedatives, RASS level and severe-pain events. 
Table 4 Incidence of serious adverse events during each phase of the study

\begin{tabular}{|c|c|c|c|c|c|c|c|}
\hline & $\begin{array}{c}\text { Phase } 1 \\
\text { (baseline) } \\
n=182 \\
\end{array}$ & $\begin{array}{c}\text { Phase } 2 \\
\text { (intervention) } \\
n=129\end{array}$ & $\begin{array}{c}\text { Phase } 3 \\
\text { (adjustment) } \\
n=170\end{array}$ & $\begin{array}{c}\text { Phase } 4 \\
\text { (consolidation) } \\
n=149 \\
\end{array}$ & $\begin{array}{c}P \\
1-2\end{array}$ & $\begin{array}{c}P \\
1-3\end{array}$ & $\begin{array}{c}P \\
1-4\end{array}$ \\
\hline Cardiac arrest, n (\%) & $2(1 \%)$ & $0(0 \%)$ & $0(0 \%)$ & $0(0 \%)$ & & & \\
\hline Arrhythmias, n (\%) & $3(2 \%)$ & $4(3 \%)$ & $1(1 \%)$ & $0(0 \%)$ & & & \\
\hline Tachycardia, n (\%) & $5(3 \%)$ & $5(4 \%)$ & $3(2 \%)$ & $6(4 \%)$ & & & \\
\hline Bradycardia, n (\%) & $0(0 \%)$ & $2(2 \%)$ & $0(0 \%)$ & $0(0 \%)$ & & & \\
\hline Hypertension, n (\%) & $14(8 \%)$ & $2(2 \%)$ & $6(4 \%)$ & $11(7 \%)$ & & & \\
\hline Hypotension, n (\%) & $8(4 \%)$ & $5(4 \%)$ & $2(1 \%)$ & $9(6 \%)$ & & & \\
\hline Oxygen desaturation, n (\%) & $19(10 \%)$ & $15(12 \%)$ & $8(5 \%)$ & $6(4 \%)$ & & & \\
\hline Bradypnea, n (\%) & $2(1 \%)$ & $6(5 \%)$ & $0(0 \%)$ & $0(0 \%)$ & & & \\
\hline Ventilatory distress, n (\%) & $24(13 \%)$ & $13(10 \%)$ & $15(9 \%)$ & $2(1 \%)$ & & & \\
\hline At least one event, n (\%) & $68(37 \%)$ & $36(28 \%)$ & $29(17 \%)$ & $31(21 \%)$ & 0.09 & $<0.001$ & 0.005 \\
\hline
\end{tabular}

Statistical analysis was performed using a generalized linear mixed-effects model for repeated measures.

appreciation of patients' pain and analgesics needs. A multidisciplinary discussion involving nurses and physicians/pharmacists is recommended regarding the complex management of pain in ICU patients [41]. To better define a rational plan for a given patient, it is important for physicians to assess nursing issues as it should be important for nurses to understand the benefit and risks associated with every analgesic ordered by physicians.

Tramadol was the only drug that's use significantly increased through the study. Except in the case of severe renal impairment, tramadol is an opioid associated with a minimal risk of ventilatory depression [42]. This could explain the preference of the team regarding its use in ICU patients who are at high risk of ventilatory depression. Similarly, tramadol use significantly increased in a previous quality improvement project aimed at reducing pain at rest in ICU patients [1]. In that study, incidence of pain significantly decreased through the quality improvement project as well as the duration of mechanical ventilation [1]. Similarly, in the present study, incidence of severe pain decreased as analgesic drug use increased without any increase of SAE. That could be attributed to an accurate evaluation of the benefit:risk ratio associated with analgesic ordering. Also, the incidence of moderate pain did not significantly decrease throughout the study. Actually, complete suppression of pain could be difficult or impossible in ICU patients considering the pain syndromes (surgery, trauma, acute pancreatitis) or contraindication of analgesic drugs in critical-illness (acetaminophen and liver dysfunction, anti-inflammatory drugs and renal dysfunction). In this

Table 5 Proportion of pharmacological and non-pharmacological therapies used during each phase of the study

\begin{tabular}{|c|c|c|c|c|c|c|c|}
\hline & $\begin{array}{c}\text { Phase } 1 \\
\text { (baseline) } \\
n=184 \\
\end{array}$ & $\begin{array}{c}\text { Phase } 2 \\
\text { (intervention) } \\
n=129 \\
\end{array}$ & $\begin{array}{c}\text { Phase } 3 \\
\text { (adjustment) } \\
n=170\end{array}$ & $\begin{array}{c}\text { Phase } 4 \\
\text { (consolidation) } \\
n=149 \\
\end{array}$ & $\begin{array}{l}P \\
1-2\end{array}$ & $\begin{array}{l}P \\
1-3\end{array}$ & $\begin{array}{l}P \\
1-4\end{array}$ \\
\hline \multicolumn{8}{|l|}{ Analgesics drugs, n (\%) } \\
\hline WHO step 3 & $28(15 \%)$ & $32(25 \%)$ & $36(21 \%)$ & $33(22 \%)$ & 0.11 & 0.14 & 0.12 \\
\hline WHO step 2: tramadol & $17(9 \%)$ & $25(19 \%)$ & $48(28 \%)$ & $49(33 \%)$ & 0.19 & 0.001 & $<0.001$ \\
\hline WHO step 1: acetaminophen & $29(16 \%)$ & $23(18 \%)$ & $44(26 \%)$ & $36(24 \%)$ & 0.72 & 0.23 & 0.17 \\
\hline nefopam & $22(12 \%)$ & $9(7 \%)$ & $26(15 \%)$ & $33(22 \%)$ & 0.24 & 0.71 & 0.11 \\
\hline At least one drug & $60(33 \%)$ & $56(43 \%)$ & $86(51 \%)$ & $79(53 \%)$ & 0.22 & 0.01 & 0.002 \\
\hline Number of drugs per patient, mean (SD) & $0.52(0.70)$ & $0.69(0.77)$ & $0.91(0.85)$ & $1.01(0.97)$ & 0.30 & 0.008 & $<0.001$ \\
\hline \multicolumn{8}{|l|}{ Non pharmacological therapies } \\
\hline Explication*, n (\%) & $158(87 \%)$ & $91(71 \%)$ & $140(82 \%)$ & & 0.01 & 0.62 & ND \\
\hline Massage, n (\%) & $120(66 \%)$ & $82(64 \%)$ & $64(38 \%)$ & & 0.86 & $<0.001$ & ND \\
\hline Standard music listening, n (\%) & $12(7 \%)$ & $10(8 \%)$ & $4(2 \%)$ & & 0.08 & $<0.001$ & ND \\
\hline Music therapy, n (\%) & $0(0 \%)$ & $5(4 \%)$ & $0(0 \%)$ & & 0.99 & 1.00 & ND \\
\hline At least one therapy, n (\%) & $160(88 \%)$ & $98(76 \%)$ & $142(84 \%)$ & & 0.06 & 0.54 & ND \\
\hline Number of therapies per patient, mean (SD) & $2(1 ; 3]$ & $3(1 ; 4]$ & $1(1 ; 2]$ & & $<0.01$ & 0.05 & ND \\
\hline
\end{tabular}

ND, not done (external audit of medical chart records); SD, Standard Deviation; WHO, World Health Organization. Analgesics were classified according to the WHO's pain relief ladder [55] used to treat pain. $1^{\text {st }}$ step, non-opioid analgesics; $2^{\text {nd }}$ step, minor opioids; $3^{\text {rd }}$ step, major opioids. Non-pharmacological therapies were evaluated for the three first phases. Non-pharmacological therapies were not assessed during the post-intervention Phase 4 (see text). ${ }^{*}$ Explication of the procedure process, insurance that pain will be taken into consideration, if any. 
way, American guidelines recommended defining an acceptable threshold of pain according to the context for each patient [43].

In order to reduce the risk of such drug adverse events, non-pharmacological therapies were developed throughout the study. Despite the implementation of music therapy as a new technology available for every patient and despite specific educational interventions, there was an increased use of non-pharmacological therapy but this increase was not sustained during the third phase of the study. Music therapy was poorly implemented throughout the project. Some nurses reported that the time which was required for a music therapy session (40 minutes) did not allow for easily preventing or treating procedural pain contrary to analgesic drugs. Also, nurses should have been more comfortable with analgesic drug use as the quality-improvement project was developed and might have discarded non-pharmacological therapies at the same time for different reasons including trust in their efficacy, timing and so on. If positive effects of music therapy and standard music listening have been shown in small-sized physiological studies in critically ill patients $[44,45]$, the feasibility and impact of larger routine implementation has yet to be evaluated. Moreover, obstacles to widespread use of non-pharmacological therapy rather than analgesic drugs need to be explored because the rationale for development of non-pharmacological therapy in critical care is strong.

Decreased pain-associated stress response could partly explain the decrease of SAE observed during the last two studied phases (adjusted intervention and consolidation P-D-C-A-steps). Pain induces reflex responses that may alter respiratory mechanics and increase cardiac demand via tachycardia and increased myocardial oxygen consumption, leading to desaturation and blood pressure changes. Stress response may also induce hypercoagulability, immunosuppression and persistent catabolism $[4,5]$. In the present study, multivariate analysis adjusted to cofactors, such as severity of illness showed that severe pain events were an independent risk factor of SAE. This was confirmed by the sensitivity analysis, removing the most common pain-related adverse events (tachycardia and hypertension). Use of analgesics may decrease stress response in critically ill patients [46,47]. In our study, the main SAE observed were oxygen desaturation and ventilator distress (Table 4). The rate of these SAE decreased throughout the study, although ventilator management or oxygenation practices were not changed, contrary to pain management practices.

This study constitutes an improvement in quality and safety in healthcare. Such processes are fundamental to improving our healthcare, by changing our systems, avoiding overuse of ineffective care and underuse of effective care [48]. Quality improvement methods, such as the Plan-Do-Check-Adjust cycle, seek to apply proven treatments and recommended strategies to "real world" patients, allowing the integration of "best evidence" and "clinical evidence" [20,22]. To our knowledge, there are no published data regarding the feasibility of a quality improvement process for moving ICU patients. Changing practices is challenging in an ICU setting, with necessary education of a large team $[49,50]$. Moreover, a multidisciplinary approach is essential, placing responsibility with the team rather than with individuals. Differences in pain appreciation among physicians, nurses and assistant nurses are well known in the ICU setting [34] and were found again in our questionnaire. It has been previously reported that ICU physicians under-evaluated patients' pain compared to nurses [51], and that ICU nurses under-evaluated patients' pain compared to assistant nurses [52].

Our study has several limitations. First, there were less missing data in the third phase (adjusted intervention PD-C-A phase) than in the two first phases. This could be explained by a high workload during February and September 2010, much higher than in April 2011. Indeed, one-third of the unit had to be closed unexpectedly in April after Phase 3 had begun. To deal with missing data and to avoid a possible bias due to more frequently evaluating patients in pain in the two first phases, patients were randomly enrolled in Phase 4. This phase (consolidation of P-D-C-A-steps) was aimed to reinforce the results observed in the previous phase $[21,23]$. Second, pain was evaluated by the bedside $R N$ (BPS) or by the patient with the help of the bedside RN (NRS), and not by an independent investigator. However, this design is appropriate in a quality improvement process of routine care because self-evaluation of the caregiver is part of the improvement process $[22,53]$. Moreover, even if it was not possible to have an independent investigator at the bedside for all 16 patients during the turning every morning, the presence of an observer could have introduced another bias leading to more accurate care [32]. In this way, the study sheets were de-identified regarding the $\mathrm{RN}$ to allow for more independent evaluation of care. Also, the study design requires including all consecutive turnings within one month to deal with a possible punctual Hawthorne effect and to transform it in an acquired routine process [32]. The findings of this quality study can be supported by the incidence of SAE, which were objectively evaluated and also decreased along with the incidence of severe pain through the study. Third, if the global impact of educational interventions was supported by a decreased incidence of pain and SAE along with an increased rate of analgesic administration, no qualitative 
method was performed to better assess the impact of each aspect of educational interventions on health caregivers' skill regarding pain management as well as nurse-physician interaction and nurse autonomy [14,54]. Finally, pain management during other nursing and medical procedures (tracheal suctioning, central intravenous line placement...) was not evaluated. This should be a further step in our quality improvement project.

\section{Conclusions}

A focused quality improvement project on pain management in the ICU was associated with improved pain management during patient turning for nursing procedures as determined by 1 ) a decreased incidence of severe pain; 2) an increased use of analgesic drugs; 3 ) a decreased incidence of serious adverse events. Careful documentation of pain management while moving ICUpatients for nursing procedures could be implemented as a health quality indicator in the ICU-setting.

\section{Key messages}

- Moving an ICU patient for nursing care procedures is associated with serious adverse events in one out of three procedures.

- Serious adverse events are strongly associated with severe pain during these procedures.

- Health quality improvement of pain management is associated with a decrease of both severe pain and serious adverse events.

- Careful documentation of pain management while moving ICU patients could be implemented as a health quality indicator in the ICU setting.

\section{Additional material}

Additional file 1: Analgesia protocol for procedures - English language. Poster referring to procedural pain management, created by the work group to highlight educational objectives and posted in every patient's room. English translation.

Additional file 2: Algorithm for continuous sedation-analgesia English language. Poster referring to continuous sedation-analgesia algorithm, adapted from [18] by the work group to highlight educational objectives and posted in every patient's room. English translation.

Additional file 3: Analgesia protocol for procedures - French language. Poster referring to procedural pain management, created by the work group to highlight educational objectives and posted in every patient's room. Original French version.

Additional file 4: Algorithm for continuous sedation-analgesia French language. Poster referring to continuous sedation-analgesia algorithm, adapted from [18] by the work group to highlight educational objectives and posted in every patient's room. Original French version.

Additional file 5: Additional tables. Table S1: Incidence of pain calculated on overall procedures for each of the four studied phases. Table S2: Sensitivity analysis of factors associated with serious adverse events determined by multivariate mixed-effects model analysis after removing tachycardia and/or hypertension from serious adverse events.

\section{Abbreviations}

BPS: Behavioral pain scale; BPS-NI: non-intubated BPS; COGAR: Comité d'Organisation et de Gestion de I'Anesthésie Réanimation du Centre Hospitalier Universitaire de Montpellier; ICU: Intensive care unit; NRS: Numeric rating scale; RASS: Richmond Agitation Sedation Scale; RN: Registered nurse; SAE: Serious adverse event; SAPS: Simplified Acute Physiological Score; WHO: World Health Organization

\section{Authors' contributions}

GC, SJ, SDL, CG and MPS conceived the study and participated in its design and coordination. ADJ and NM performed the statistical analysis. ADJ, GC and SJ drafted the manuscript. MC and BJ helped to correct the manuscript. $\mathrm{JC}$ was in charge of English editing. All authors read and approved the final manuscript.

\section{Competing interests}

The authors declare that they have no competing interests.

\section{Acknowledgements}

The authors are grateful for the enthusiastic support of ICU nurses, assistant nurses, residents and physicians at University of Montpellier Saint Eloi Hospital. They thank the Hospital Pain Committee and the Department of Communication at University of Montpellier Hospitals, especially Ms. Gaëlle Faugier for her very helpful contribution to educational supports.

\section{Author details}

'Intensive Care and Anesthesiology Department, University of Montpellier Saint Eloi Hospital, 80 Avenue Augustin Fliche, Montpellier, 34295, France. ${ }^{2}$ Department of Statistics, University of Montpellier Lapeyronie Hospital, 39 Avenue Charles Flahaut, Montpellier, 34295, France. ${ }^{3}$ Unité U1046 de l'Institut National de la Santé et de la Recherche Médicale (INSERM), Arnaud de Villeneuve Hospital, University of Montpellier 1, University of Montpellier 2, 39 Avenue Charles Flahaut, Montpellier, 34295, France.

Received: 26 October 2012 Revised: 15 January 2013

Accepted: 25 February 2013 Published: 18 April 2013

\section{References}

1. Chanques G, Jaber S, Barbotte E, Violet S, Sebbane M, Perrigault P, Mann C, Lefrant J, Eledjam J: Impact of systematic evaluation of pain and agitation in an intensive care unit. Crit Care Med 2006, 34:1691-1699.

2. Chanques G, Sebbane M, Barbotte E, Viel E, Eledjam JJ, Jaber S: A prospective study of pain at rest: incidence and characteristics of an unrecognized symptom in surgical and trauma versus medical intensive care unit patients. Anesthesiology 2007, 107:858-860.

3. Payen JF, Chanques G, Mantz J, Hercule C, Auriant I, Leguillou JL, Binhas M, Genty C, Rolland C, Bosson JL: Current practices in sedation and analgesia for mechanically ventilated critically ill patients: a prospective multicenter patient-based study. Anesthesiology 2007, 106:687-695.

4. Lewis KS, Whipple JK, Michael KA, Quebbeman EJ: Effect of analgesic treatment on the physiological consequences of acute pain. Am J Hosp Pharm 1994, 51:1539-1554.

5. Epstein J, Breslow M: The stress response of critical illness. Crit Care Clin 1999, 15:17-33.

6. Robinson BR, Mueller EW, Henson K, Branson RD, Barsoum S, Tsuei BJ: An analgesia-delirium-sedation protocol for critically ill trauma patients reduces ventilator days and hospital length of stay. J Trauma 2008, 65:517-526.

7. Payen JF, Bosson JL, Chanques G, Mantz J, Labarere J, DOLOREA Investigators: Pain assessment is associated with decreased duration of mechanical ventilation in the intensive care unit: a post hoc analysis of the DOLOREA study. Anesthesiology 2009, 111:1308-1316.

8. Skrobik Y, Ahern S, Leblanc M, Marquis F, Awissi DK, Kavanagh BP: Protocolized intensive care unit management of analgesia, sedation, and delirium improves analgesia and subsyndromal delirium rates. Anesth Analg 2010, 111:451-463.

9. Puntillo KA, Wild LR, Morris AB, Stanik-Hutt J, Thompson CL, White C: Practices and predictors of analgesic interventions for adults undergoing painful procedures. Am J Crit Care 2002, 11:415-429. 
10. Martin J, Franck M, Sigel S, Weiss M, Spies C: Changes in sedation management in German intensive care units between 2002 and 2006: a national follow-up survey. Crit Care 2007, 11:R124

11. Reschreiter $H$, Maiden M, Kapila A: Sedation practice in the intensive care unit: a UK national survey. Crit Care 2008, 12:R152.

12. Schweickert WD, Kress JP: Strategies to optimize analgesia and sedation. Crit Care 2008, 12(Suppl 3):S6.

13. Puntillo K, Morris A, Thompson C, Stanik-Hutt J, White C, Wild L: Pain behaviors observed during six common procedures: results from Thunder Project II. Crit Care Med 2004, 32:421-427.

14. Subramanian $P$, Allcock $N$, James $V$, Lathlean J: Challenges faced by nurses in managing pain in a critical care setting. J Clin Nurs 2012, 21:1254-1262.

15. Bertolini G, Minelli C, Latronico N, Cattaneo A, Mura G, Melotti R, lapichino G, Gruppo Italiano per la Valutazione degli Interventi in Terapia Intensiva: The use of analgesic drugs in postoperative patients: the neglected problem of pain control in intensive care units. An observational, prospective, multicenter study in 128 Italian intensive care units. Eur J Clin Pharmacol 2002, 58:73-77.

16. Vazquez M, Pardavila MI, Lucia M, Aguado Y, Margall MA, Asiain MC: Pain assessment in turning procedures for patients with invasive mechanical ventilation. Nurs Crit Care 2011, 16:178-185.

17. Constantin JM, Leone M, Jaber S, Allaouchiche B, Orban JC, Cannesson M, Fourcade O, Morel J, Martin C, Lefrant JY, AzuRéa: Activity and the available human resources working in 66 French Southern intensive care units. Ann Fr Anesth Reanim 2010, 29:512-517.

18. Dodek P, Chanques G, Brown G, Norena M, Grubisic M, Wong H, Jaber S: Role of organisational structure in implementation of sedation protocols: a comparison of Canadian and French ICUs. BMJ Qual Saf 2012, 21:715-721.

19. Sauder P, Andreoletti M, Cambonie G, Capellier G, Feissel M, Gall O, Goldran-Toledano D, Kierzek G, Mateo J, Mentec H, Mion G, Rigaud JP, Seguin P: Sedation and analgesia in intensive care (with the exception of new-born babies). Ann Fr Anesth Reanim 2008, 27:541-551.

20. Brattebø G, Hofoss D, Flaatten H, Muri AK, Gjerde S, Plsek PE: Effect of a scoring system and protocol for sedation on duration of patients' need for ventilator support in a surgical intensive care unit. BMJ 2002, 324:1386-1389.

21. Deming W: Principles for transformation. Out of the Crisis Cambridge, MA: MIT Press; 1986, 18-96.

22. Berwick DM: Developing and testing changes in delivery of care. Ann Intern Med 1998, 128:651-656.

23. Ishikawa K: What is Total Quality Control? The Japanese Way Englewood Cliffs, NJ: Prentice-Hall, Inc; 1985, 56-61.

24. Chanques G, Viel E, Constantin JM, Jung B, de Lattre S, Carr J, Cissé M, Lefrant JY, Jaber $S$ : The measurement of pain in intensive care unit: comparison of 5 self-report intensity scales. Pain 2010, 151:711-721.

25. Payen JF, Bru O, Bosson JL, Lagrasta A, Novel E, Deschaux I, Lavagne P, Jacquot C: Assessing pain in critically ill sedated patients by using a behavioral pain scale. Crit Care Med 2001, 29:2258-2263.

26. Chanques G, Payen JF, Mercier G, de Lattre S, Viel E, Jung B, Cissé M, Lefrant JY, Jaber $S$ : Assessing pain in non-intubated critically ill patients unable to self report: an adaptation of the Behavioral Pain Scale. Intensive Care Med 2009, 35:2060-2067.

27. Hamill-Ruth RJ, Marohn ML: Evaluation of pain in the critically ill patient. Crit Care Clin 1999, 15:35-54.

28. Young J, Siffleet J, Nikoletti S, Shaw T: Use of a Behavioural Pain Scale to assess pain in ventilated, unconscious and/or sedated patients. Intensive Crit Care Nurs 2006, 22:32-39.

29. Sessler CN, Gosnell MS, Grap MJ, Brophy GM, O'Neal PV, Keane KA, Tesoro EP, Elswick RK: The Richmond Agitation-Sedation Scale: validity and reliability in adult intensive care unit patients. Am J Respir Crit Care Med 2002, 166:1338-1344.

30. Chanques $G$, Jaber $S$, Barbotte $E$, Verdier $R$, Henriette $K$, Lefrant J, Eledjam J: Validation of the French translated Richmond vigilance-agitation scale. Ann Fr Anesth Reanim 2006, 25:696-701.

31. Legall J-R, Lemeshow S, Saulnier F: New Simplified Acute Physiology Score (SAPS II) based on a European/North American Multicenter Study. JAMA 1993, 270:2957-2963.

32. Wickstrom G, Bendix T: The "Hawthorne effect" - what did the original Hawthorne studies actually show? Scand J Work Environ Health 2000, 26:363-367.
33. Stanik-Hutt JA, Soeken KL, Belcher AE, Fontaine DK, Gift AG: Pain experiences of traumatically injured patients in a critical care setting. Am J Crit Care 2001, 10:252-259.

34. Grosclaude C, Asehnoune K, Demeure D, Millet S, Champin P, Naux E, Malinge M, Lejus C: Opinion of different professional categories about the intensity of procedural pain in adult intensive care units. $\mathrm{Ann} \mathrm{Fr}$ Anesth Reanim 2010, 29:884-888.

35. Hall JB: Creating the animated intensive care unit. Crit Care Med 2010, 38 : S668-675.

36. Denehy L, Elliott D: Strategies for post ICU rehabilitation. Curr Opin Crit Care 2012, 18:503-508.

37. Hamill-Ruth RJ: Use of analgesics in the intensive care unit: who says it hurts? Crit Care Med 2002, 30:2597-2598.

38. Gommers D, Bakker J: Medications for analgesia and sedation in the intensive care unit: an overview. Crit Care 2008, 12(Suppl 3):S4.

39. Mrozek S, Constantin JM, Futier E, Zenut M, Ghardes G, Cayot-Constantin S, Bonnard M, Ait-Bensaid N, Eschalier A, Bazin JE: Acetaminophen-induced hypotension in intensive care unit: a prospective study. Ann Fr Anesth Reanim 2009, 28:448-453.

40. Chanques G, Sebbane M, Constantin JM, Ramillon N, Jung B, Cissé M, Lefrant JY, Jaber S: Analgesic efficacy and haemodynamic effects of nefopam in critically ill patients. Br J Anaesth 2011, 106:336-343.

41. Marshall J, Finn CA, Theodore AC: Impact of a clinical pharmacistenforced intensive care unit sedation protocol on duration of mechanical ventilation and hospital stay. Crit Care Med 2008, 36:427-433.

42. Barnung SK, Treschow M, Borgbjerg FM: Respiratory depression following oral tramadol in a patient with impaired renal function. Pain 1997, 71:111-112.

43. Jacobi J, Fraser GL, Coursin DB, Riker RR, Fontaine D, Wittbrodt ET, Chalfin DB, Masica MF, Bjerke HS, Coplin WM, Crippen DW, Fuchs BD, Kelleher RM, Marik PE, Nasraway SA, Murray MJ, Peruzzi WT, Lumb PD: Clinical practice guidelines for the sustained use of sedatives and analgesics in the critically ill adult. Crit Care Med 2002, 30:119-141.

44. Jaber S, Bahloul H, Guétin S, Chanques G, Sebbane M, Eledjam JJ: Effects of music therapy in intensive care unit without sedation in weaning patients versus non-ventilated patients. Ann Fr Anesth Reanim 2007, 26:30-38.

45. Conrad C, Niess H, Jauch KW, Bruns CJ, Hartl W, Welker L: Overture for growth hormone: requiem for interleukin-6? Crit Care Med 2007, 35:2709-2713.

46. Mangano D, Siliciano D, Hollenberg M, Leung J, Browner W, Goehner P, Merrick S, Verrier E: Postoperative myocardial ischemia. Therapeutic trials using intensive analgesia following surgery. The Study of Perioperative Ischemia (SPI) Research Group. Anesthesiology 1992, 76:342-353.

47. Parker S, Breslow M, Frank S, Rosenfeld B, Norris E, Christopherson R, Rock P, Gottlieb S, Raff H, Perler B, et al: Catecholamine and cortisol responses to lower extremity revascularization: correlation with outcome variables. Perioperative Ischemia Randomized Anesthesia Trial Study Group. Crit Care Med 1995, 23:1954-1961.

48. Committee on Quality of Health Care in America, Institute of Medicine: Crossing the Quality Chasm: A New Health System for the 21st Century Washington, DC: National Academy Press; 2001.

49. Jain M, Miller L, Belt D, King D, Berwick DM: Decline in ICU adverse events, nosocomial infections and cost through a quality improvement initiative focusing on teamwork and culture change. Qual Saf Health Care 2006, 15:235-239.

50. Curtis JR, Nielsen EL, Treece PD, Downey L, Dotolo D, Shannon SE, Back AL, Rubenfeld GD, Engelberg RA: Effect of a quality-improvement intervention on end-of-life care in the intensive care unit: a randomized trial. Am J Respir Crit Care Med 2011, 183:348-355.

51. Whipple J, Lewis K, Quebbeman E, Wolff M, Gottlieb M, Medicus-Bringa M, Hartnett K, Graf M, Ausman R: Analysis of pain management in critically ill patients. Pharmacotherapy 1995, 15:592-599.

52. Hall-Lord M, Larsson G, Steen B: Pain and distress among elderly intensive care unit patients: comparison of patients' experiences and nurses' assessments. Heart Lung 1998, 27:123-132.

53. Kirschenbaum L, Kurtz S, Astiz M: Improved clinical outcomes combining house staff self-assessment with an audit-based quality improvement program. J Gen Intern Med 2010, 25:1078-1082.

54. Broyles LM, Colbert AM, Tate JA, Swigart VA, Happ MB: Clinicians' evaluation and management of mental health, substance abuse, and 
chronic pain conditions in the intensive care unit. Crit Care Med 2008, 36:87-93.

55. The American Pain Society: Principles of Analgesics Use in the Treatment of Acute Pain and Cancer Pain. 4 edition. Glenview, IL: American Pain Society; 1999.

doi:10.1186/cc12683

Cite this article as: de Jong et al:: Decreasing severe pain and serious adverse events while moving intensive care unit patients: a prospective interventional study (the NURSE-DO project). Critical Care 2013 17:R74.

Submit your next manuscript to BioMed Central and take full advantage of:

- Convenient online submission

- Thorough peer review

- No space constraints or color figure charges

- Immediate publication on acceptance

- Inclusion in PubMed, CAS, Scopus and Google Scholar

- Research which is freely available for redistribution

Submit your manuscript at www.biomedcentral.com/submit 